\title{
Desarrollo local y gobiernos locales de Ancash 2010-2014: una aproximación a su desempeño
}

Local development and local governments of Ancash 2010-2014: an approach to its performance

Guillermo Peláez Díaz ${ }^{1}$, Jorge Castillo Picón ${ }^{1}$ y Jorge Manrique Cáceres ${ }^{1}$

\section{RESUMEN}

El objetivo del presente trabajo de investigación ha sido, determinar el nivel de participación de los gobiernos municipales provinciales de Ancash, en la generación del desarrollo local, usando para tal fin el indicador de Calidad de Desempeño Local. El tipo de investigación usado para tal fin es cuantitativa, el diseño de investigación es no experimental, de corte transversal y descriptivo. La medición del desempeño municipal se ha realizado con el índice de calidad de desempeño local propuesto por Huther y Anwar (2006) cuyos índices compuestos son: a) asegurar la transparencia y la vocería de todos los ciudadanos; b) ofrecer servicios públicos y eficientes; c) promover la salud y el bienestar de los ciudadanos y d) crear un clima favorable para el crecimiento económico estable. Los resultados muestran que el Índice de calidad de desempeño municipal de los concejos provinciales de Ancash está en el rango de 27 a 40, indicando que los gobiernos locales provinciales, no contribuyen actualmente a la generación de desarrollo local.

Palabras clave: desempeño municipal; desarrollo local; calidad de servicio.

\begin{abstract}
The objective of this research was to determine the level of provincial municipality governments of Ancash participation in the generation of local development, using for this purpose the quality indicator Local Performance. The type of research used for this purpose is quantitative research design is not experimental, cross-sectional
\end{abstract}

1 Universidad Nacional Santiago Antúnez de Mayolo. Huaraz, Perú. 
and descriptive Municipal performance measurement was performed with local quality index performance proposed by Huther and Anwar (2006) with composite indexes they are: a) ensure transparency and spokesman for all citizens; b) provide public and efficient services; c) promote health and well-being of citizens and d) creating a favorable climate for stable economic growth.

The results show that the quality index of municipal performance of provincial councils of Ancash is in the range of 27 to 40, indicating that the provincial local governments, not currently contribute to the generation of local development.

Keywords: municipal performance; local development; quality of service.

\section{ICHIKLLACHAW}

Kay musyapakuywanqa riqitsimantsik imanaw Anqash markakunachaw pushaqninkuna uryayanqantam, tsaywan imanaw kay markakuna wiñayanqantam. Tsaypaq kwantitatiwawan mana ikpirimintal musyapakuywanmi rurakashqa, hinaman transwirsal nishqan willakuyninwan.

Kay munisipalkunapa alli rurayninkunataqa, shumaq rurayninkunawanmi tupukashqa, kaytaqa Hutherwan Anwarmi (2006) kamayarqan. Kaykuna kayan: a) tsuya rurayninkuna, hinaman kikin taakuq runakuna niyanqankuna; b) llapan taakuq runakunata alli yanapaynin; c) llapan taakuq runakunata alli sirwiynin, d) Alli qillayyuq imaypis kayaananpaq.

Kay musyapakuyqa Anqash munisipalkunachaw alli rurayninkuna kanqantam rikaatsimantsik, maa 27piq 40kamallam alli rurayninkuna chan. Kay markakunapa pushaqninkuna alli rurayninkuna manam alliqllaqa rikakantsu, manam alli wiñayninkuna kantsu.

Pushaq shimikuna: munisipalkunapa alli ruraynin; kikin markapa alli wiñaynin; runakunata alli yanapaynin.

\section{INTRODUCCIÓN}

El rol del municipio en el desarrollo local está vinculado a su participación activa en el desarrollo económico a través de aspectos como el redimensionamiento funcional, la promoción del mejoramiento de los recursos humanos, las actividades vinculadas con la prestación de servicios de salud, las relacionadas con el urbanismo local y la participación en la formulación de los planes de desarrollo nacional. Por tanto, el de- 
sarrollo local impulsado desde el municipio es una estrategia clave para la explotación de las capacidades que existen en la localidad.

El objetivo de la presente investigación es determinar el nivel de participación de los gobiernos municipales provinciales de Ancash en la generación del desarrollo local, para lo cual se usa el indicador de calidad de desempeño local propuesto por Huther, que mide la transparencia y la vocería de todos los ciudadanos; la oferta de servicios públicos y su eficiencia; la promoción de la salud y el bienestar de los ciudadanos y la creación de un clima favorable para el crecimiento económico estable. Actualmente, la evaluación del desempeño local es limitada; por lo general está orientada a medir la capacidad de gasto, el cumplimiento de metas de programas sociales; pero aún no existe un sistema de medición global del desempeño municipal, cuyos resultados permitan propuestas de política social. El trabajo de investigación contribuye a medir esta falencia de indicadores en la gestión municipal.

Las investigaciones con relación al tema indican que la mayoría de los municipios de Ancash, han gastado sus recursos financieros, sin haber definido un plan integral de desarrollo para su localidad y sin haber definido un perfil sustentable para la producción. Por otro lado, no existen indicadores de gestión que permitan medir la participación municipal en el desarrollo local. Al respecto, Aghón (2006) sostiene que el desarrollo local se mide por la expansión de la capacidad productiva, la elevación de la productividad y de ingresos por persona, por la democratización en la toma de decisiones y el poder en general, todo ello orientado prioritariamente al mejoramiento de las condiciones de vida de los miembros de cada sociedad local; así mismo, Monroy (2003) señala que en las estrategias de desarrollo local juega un papel clave el gobierno local, como actor político fundamental, y esto, en la medida en que encarna el rol de promotor y concertador de ideas, intereses y acciones. Para absorber las nuevas funciones, especialmente las relacionadas a crear las condiciones para promover el desarrollo, los municipios requieren tanto de recursos técnicos como de financieros. Por otro lado, Vásquez (2001), sostiene que el desarrollo económico local es un proceso de crecimiento y cambio estructural de la economía de una ciudad, comarca o región, en que se puede identificar al menos tres dimensiones: una económica, caracterizada por su sistema de producción que permite a los empresarios locales usar eficientemente los factores productivos, generar economías de escala y aumentar la productividad a niveles que permitan la competitividad en los mercados; otra sociocultural, en la cual el sistema de relaciones económicas y sociales, las instituciones locales y los valores sirven de base al proceso de desarrollo; y otra política y administrativa, en la que las iniciativas locales crean un entorno favorable a la producción e impulsan el desarrollo. 
En el Perú, los indicadores para medir la gestión municipal son muy limitados, contrariamente a lo que ocurre en los países de Europa, Canadá y E.U., donde los municipios son evaluados a través de indicadores sociales y de gestión.

La teoría usada en la investigación es la basada en la competitividad de las áreas locales de Porter (2000), que señala que existe una serie de factores que inciden en el desarrollo económico y local de un área geográfica, entre ellos señala a los recursos humanos y naturales, la dotación de infraestructura, los recursos financieros y de capitales; y el medio ambiente; el entorno macroeconómico de las empresas residentes en las áreas locales; los factores relacionados a las empresas y la organización/configuración industrial donde ellas compiten y las acciones e interacciones de los agentes que inciden en el desarrollo económico de un área geográfica. Para medir el desempeño local se usa el indicador propuesto por Hunther y Shah (2008), que consta de cuatro índices compuestos: a) asegurar la transparencia y la vocería de todos los ciudadanos; b) ofrecer servicios públicos y eficientes; c) promover la salud y el bienestar de los ciudadanos y d) crear un clima favorable para el crecimiento económico estable. Los resultados son medidos con el índice de gobernabilidad de la siguiente manera: Buena gobernabilidad; gobernabilidad media y gobernabilidad pobre.

Para el presente estudio, algunos de estos indicadores han sido adaptados al contexto del gobierno local. El índice de participación ciudadana se compone del índice que evalúa el grado de libertad política con el que se valora la capacidad de los ciudadanos de influir en la calidad de gobernabilidad que reciben. La orientación de los gobiernos hacia la provisión de bienes y servicios públicos se evalúa mediante la eficiencia burocrática y ausencia de corrupción estos se basan en encuestas que intentan calibrar el grado en que los empleados del sector público se concentran en servir al pueblo en lugar de enriquecerse a sí mismos o a sus partidos políticos.

El desarrollo social se evalúa mediante el índice de desarrollo de las Naciones Unidas (IDH) y los coeficientes de Gini (que cuantifican el grado de desigualdad del ingreso). El IDH cuantifica expectativas de vida, niveles de educación promedio e ingreso per cápita. Los coeficientes de Gini se basan en encuestas sobre distribución del ingreso.

La calidad de gestión económica se evalúa mediante indicadores de desempeño fiscal (razón de presupuesto institucional modificado a presupuesto ejecutado), libertad de decisión de gasto en relación a la Dirección General de Presupuesto Público (razón de proyectos aprobados por la municipalidad a proyectos totales). El índice de orientación hacia el exterior incluye el componente de percepción del inversionista sobre la receptividad de un gobierno al comercio, a la empresa. 


\section{MATERIALES Y METODOS}

El tipo de estudio es cuantitativo, el diseño empleado es no experimental de corte transversal y descriptivo; la población analizada estuvo conformada por los municipios provinciales de Ancash 2010-2014; la muestra empleada ha sido censal; es decir, se ha incluido en el análisis al total de los municipios provinciales; la selección de las municipalidades para el estudio, ha estado basada en la categoría de municipios provinciales reconocidos por el directorio nacional de municipalidades del Perú; los datos usados de las municipalidades son de carácter público, por lo que no se ha solicitado el conocimiento informado. La investigación se ha realizado en gabinete, los ambientes físicos usados pertenecen a la Escuela de Economía de la Universidad Nacional «Santiago Antúnez de Mayolo». La información usada en la investigación ha sido de carácter secundario, la recopilación ha sido a través de la guía de observación documental, las fuentes de información usadas son las siguientes: para calcular el índice de participación ciudadana se ha empleado la información estadística de la Oficina Nacional de Procesos Electorales (ONPE), a nivel provincial, correspondiente al año 2014 (últimas elecciones municipales en el Perú) en la que se muestran los resultados de la participación ciudadana en las elecciones municipales. Los datos para calcular el índice de orientación gubernamental compuesto por los índices de corrupción y por la eficiencia burocrática han sido obtenidos de las encuestas que realiza el INEI (2014) a través de la Encuesta Nacional de Hogares (ENAHO) en su sección de gobernabilidad, democracia y transparencia. El índice de desarrollo social compuesto por el Índice de desarrollo Humano y Distribución Equitativa del Ingreso ha sido calculado con las estadísticas del IDH proporcionadas por el programa de las Naciones Unidas, PNUD, y la información obtenida para la distribución del ingreso (Coeficiente de Gini) ha sido obtenida de las estadísticas del INEI 2010-2014. Los datos usados para el cálculo del índice de gestión económica han sido obtenidos del SIAF amigable del Ministerio de Economía del Perú en forma anual, tanto para la recaudación de ingresos propios como para la ejecución presupuestal.(MEF, 2014). Luego de haber obtenido los datos, estos han sido clasificados de acuerdo a las variables consideradas en el modelo de análisis de desempeño local propuesto por Huther, los datos han sido medidos a través de índices y razones, los datos obtenidos no han sido sometidos a validación y confiabilidad dado que las instituciones que las proporcionan realizan el protocolo indicado. El modelo matemático empleado para valorar el desempeño municipal, ha sido el propuesto por Huther y Shah en su artículo «una medida simple de buen gobierno». El modelo matemático es el siguiente: 


$$
\mathrm{ID}=\mathrm{CP} \infty_{1} * \mathrm{GO} \infty_{2} * \mathrm{SD}_{3} * \mathrm{EM}^{1}-\infty_{1}-\infty_{2}-\infty_{3}
$$

Donde:

$\mathrm{CP}=\mathrm{PF}^{\beta}$

$\mathrm{GO}=\mathrm{RT}^{\mathrm{K}}{ }_{1} * \mathrm{CO}^{\mathrm{K}}$

$\mathrm{SD}=\mathrm{HD}^{\mathrm{Y}} * \mathrm{GI}^{1-\mathrm{Y}}$,

$\mathrm{EM}=00_{1}^{\mathrm{M}} * \mathrm{CB}_{2}^{\mathrm{M}} * \mathrm{DB}^{1-\mathrm{M}_{1}-\mathrm{M}_{2}}$

$\infty, \beta, K, Y, M$ son ponderaciones que indican la importancia relativa de los componentes en la evaluación general del gobierno.

Tabla 1. Componentes de los índices

Nombre del índice

CP Índice de participación ciudadana

GO Índice de orientación gubernamental

SD Índice de desarrollo social

EM Índice de gestión económica
Índices componentes

PF Libertad política

RT Eficiencia burocrática

CO Ausencia de corrupción

HD Desarrollo humano

Gi Distribución equitativa del ingreso

OO Recaudación de ingresos

CB Independencia del municipio

DB Razón PIM/Presupuesto ejecutado

Estadísticamente, los datos han sido analizados usando la estadística descriptiva, la media ha sido calculada para un nivel de confianza del 95\%, la variabilidad ha sido medida con la desviación estándar, el valor de significancia aceptado ha sido medido con el coeficiente de correlación del modelo.

Los valores de los indicadores del desempeño municipal para medir la participación en el desarrollo local son: buen gobierno 51-100; gobernabilidad media 41-50 y gobernabilidad pobre $20-40$.

Los resultados obtenidos han servido para obtener la discusión y las conclusiones. 


\section{RESULTADOS}

1. Resultados estadísticos

Tabla 2. Índices parciales por municipalidades

\begin{tabular}{|c|c|c|c|c|}
\hline Municipalidad & $\begin{array}{l}\text { Índice de } \\
\text { participa- } \\
\text { ción ciuda- } \\
\text { dana }\end{array}$ & $\begin{array}{l}\text { Índice de } \\
\text { orientación } \\
\text { guberna- } \\
\text { mental }\end{array}$ & $\begin{array}{l}\text { Índice de } \\
\text { desarrollo } \\
\text { social }\end{array}$ & $\begin{array}{l}\text { Índice de ges- } \\
\text { tión económica }\end{array}$ \\
\hline & $\mathrm{CP}^{\wedge} \mathrm{a} 1$ & $\mathrm{GO}^{\wedge} \mathrm{a} 2$ & $\mathrm{SD}^{\wedge} \mathrm{a} 3$ & $E M^{\wedge} 1-a 1-a 2-a 3$ \\
\hline Huaraz & 3.05 & 2.19 & 2.55 & 2.17 \\
\hline Aija & 3.05 & 2.23 & 2.48 & 2.34 \\
\hline Antonio Raimondi & 2.99 & 2.15 & 2.48 & 2.09 \\
\hline Asunción & 3.00 & 2.19 & 2.47 & 1.93 \\
\hline Bolognesi & 3.03 & 2.24 & 2.51 & 2.34 \\
\hline Carhuaz & 3.07 & 2.17 & 2.50 & 2.06 \\
\hline Carlos F. Fitzcarrald & 3.01 & 2.24 & 2.47 & 1.82 \\
\hline Casma & 3.07 & 2.22 & 2.51 & 2.26 \\
\hline Corongo & 3.04 & 2.19 & 2.51 & 2.26 \\
\hline Huari & 3.03 & 2.19 & 2.48 & 1.93 \\
\hline Huarmey & 3.07 & 2.18 & 2.51 & 2.34 \\
\hline Huaylas & 3.04 & 2.25 & 2.51 & 2.21 \\
\hline M. Luzuriaga & 3.04 & 2.19 & 2.50 & 1.93 \\
\hline Ocros & 3.06 & 2.09 & 2.47 & 2.06 \\
\hline Pallasca & 3.01 & 2.08 & 2.51 & 2.11 \\
\hline Pomabamba & 3.02 & 2.11 & 2.51 & 1.68 \\
\hline Recuay & 3.05 & 2.09 & 2.50 & 2.43 \\
\hline Santa & 3.05 & 2.19 & 2.53 & 2.45 \\
\hline Sihuas & 3.02 & 2.13 & 2.50 & 2.14 \\
\hline Yungay & 3.05 & 2.20 & 2.50 & 2.17 \\
\hline
\end{tabular}


En la tabla 2 se observa los resultados cuantitativos de los índices parciales obtenidos por cada municipalidad provincial de Ancash.

Tabla 3. Índice de Desempeño del Gobierno Local (ID)

\begin{tabular}{ll}
\hline Municipalidad & $\mathrm{ID}=\mathrm{CP} \infty^{1} \mathrm{x} \mathrm{GO}^{2} \mathrm{x} \mathrm{SD}^{3} \mathrm{x} \mathrm{EM}^{1-\infty 1-\infty 2-\infty 3}$ \\
\hline Huaraz & 36.86 \\
Aija & 39.56 \\
Antonio Raimondi & 33.28 \\
Asunción & 31.42 \\
Bolognesi & 40.08 \\
Carhuaz & 34.34 \\
Carlos F. Fitzcarrald & 30.27 \\
Casma & 38.67 \\
Corongo & 37.84 \\
Huari & 31.89 \\
Huarmey & 39.37 \\
Huaylas & 37.98 \\
M. Luzuriaga & 32.15 \\
Ocros & 32.50 \\
Pallasca & 33.39 \\
Pomabamba & 27.05 \\
Recuay & 38.81 \\
Santa & 34.40 \\
Sihuas & \\
\hline
\end{tabular}

En la tabla 3 se observa los Índices de Desempeño Municipal (ID) obtenidos al aplicar el modelo matemático propuesto por Huther y Shah (2008). 
Tabla 4. Índices promedio a nivel de municipalidades

\begin{tabular}{cccccc}
\hline $\begin{array}{c}\text { Municipalidades } \\
\text { provinciales de } \\
\text { Ancash }\end{array}$ & $\begin{array}{c}\text { Índice de } \\
\text { participación } \\
\text { ciudadana }\end{array}$ & $\begin{array}{c}\text { Índice de } \\
\text { orientación } \\
\text { gubernamental }\end{array}$ & $\begin{array}{c}\text { Índice de } \\
\text { desarrollo } \\
\text { social }\end{array}$ & $\begin{array}{c}\text { Índice de } \\
\text { gestión } \\
\text { económica }\end{array}$ & ID \\
\hline Promedio & 85.3 & 22.53 & 39.15 & 21.9 & 35.38 \\
\hline
\end{tabular}

Tabla 5. Coeficiente de correlación

\begin{tabular}{ccccc}
\hline \multicolumn{5}{c}{ Coeficiente de correlación } \\
\hline CP & GO & SD & EM & ID \\
0.91 & 0.85 & 0.87 & 0.89 & 0.88 \\
\hline
\end{tabular}

La tabla 5 muestra las correlaciones para cada variable y, a nivel general, ha sido calculada para un nivel de significancia del 0.05

\section{DISCUSIÓN}

Los resultados muestran, en términos generales, índices de desempeño de las municipalidades provinciales entre 27.05 y 41.50 que, de acuerdo a la clasificación en el trabajo de Huther y Shah, son valorados como Gobernabilidad Pobre, lo que es corroborado por el índice promedio de gobernabilidad y por el coeficiente de correlación con una puntuación de 35.38 y una correlación de 0.88 ; respectivamente, el índice de orientación gubernamental es de 22.53, el índice de desarrollo social igual a 39.15 y el índice de gestión económica igual a 21.9; los coeficientes de correlación para cada una de las variables indicadas es de $0.85,0.87$ y 0.89 , respectivamente; lo cual significan que el servicio civil de las municipalidades para impulsar el desarrollo regional es bajo; los índices de orientación gubernamental, de desarrollo social y de gestión económica señalan que los gobiernos provinciales de Ancash mantienen un nivel de ineficiencia burocrática, desarrollo humano medio, distribución inequitativa del ingreso, morosidad en la recaudación de ingresos propios y ejecución de gastos bajo; y muestran además una inadecuada priorización de las obras en relación al impulso del desarrollo regional; por otro lado el índice de participación ciudadana es de 85.3 y su correlación es de 0.91; lo cual significan que existe libertad para elegir al alcalde, sin embargo cuando esta se 
refiere a la participación del vecino para priorizar obras no funciona adecuadamente, la decisión es política.

Los municipios de la costa como Casma y Huarmey han obtenido índices de 38.67, 39.37 respectivamente. Entre los municipios de la sierra que han alcanzado los mayores índices de desempeño municipal están: Recuay 38.81, Bolognesi 40.08, Aija 39.56, Huaylas 37.98, Corongo 37.84, Huaraz 36.86, índices que están por encima de la media general. En el caso de la Municipalidad del Santa, ha obtenido un índice de 41.50 lo que permite calificarla con un desempeño de gobernabilidad media; por lo tanto, sería la única municipalidad con desempeño aceptable; los resultados concuerdan con la investigación de la Pontificia Universidad Católica del Perú (2010) cuando señala que el índice de competitividad regional de Ancash es de 42.01; con la investigación de Huther y Shah (2008), en cuanto a la valoración de la gobernabilidad; con la investigación de Qian y Weingast (1997) cuando señala que los municipios con mejor desempeño son aquellos que sostienen la productividad, los incentivos de mercado, generan infraestructura de desarrollo y logran mantener la descentralización; los resultados no concuerdan con lo postulado por Orellana, (2009), cuando señala que el desarrollo local está vinculado a su participación activa en el desarrollo económico: redimensionamiento funcional, promoción del mejoramiento de los recursos humanos, actividades vinculadas con la prestación de servicios de salud, las relacionadas con el urbanismo local y la participación en la formulación de los planes de desarrollo nacional, y tampoco con los de Díaz y Corredor (2009) cuando indican que el desarrollo local impulsado desde el municipio es una estrategia clave para la explotación de las capacidades que existen en la localidad.

Esta investigación es una de las primeras en este ámbito que muestra resultados sobre el desempeño de las municipalidades provinciales de Ancash; los resultados están acordes con lo que se observa en la realidad si se considera que el Perú tiene un índice de 48 como calidad de la Gobernabilidad del Gobierno nacional y las Municipalidades son reflejo de lo que ocurre a nivel del Gobierno Central y Regional. Por otro lado, la media que presentan los municipios en el desempeño municipal es de 35.38 y su desviación estándar es de 3.40. El trabajo servirá como base para investigaciones futuras. Los resultados alcanzados para la municipalidad de Casma, Huarmey y del Santa refuerzan parcialmente la Hipótesis. 


\section{CONCLUSIONES}

La participación de los gobiernos municipales provinciales de Ancash en la generación del desarrollo local es pobre; los índices hallados sobre el desempeño municipal varían entre 27.05 y 41.5; el promedio de desempeño a nivel de municipios es de 35.38; el modelo matemático tiene una correlación de 0.88 ; los valores hallados significan una valoración de gobernabilidad pobre; estos resultados indican que la gestión en orientación gubernamental, desarrollo social y gestión económica son bajos, todo lo que demuestra que los municipios no promueven adecuadamente el desarrollo local.

Los Índices de Calidad de Desempeño Municipal alcanzados por los municipios provinciales son los siguientes: Huaraz 36.86, Aija 39.56, Antonio Raimondi 33.28, Asunción 31.42, Bolognesi 40.08, Carhuaz 34.34, Carlos Fermín Fitzcarrald 30.27, Casma 38.67, Corongo 37.84, Huari 31.89, Huarmey 39.37, Huaylas 37.98, Mariscal Luzuriaga 32.15, Ocros 32.50, Pallasca 33.39, Pomabamba 27.05, Recuay 38.81, Santa 41.50, Sihuas 34.44 y Yungay 36.36. El promedio es de 35.38. Los valores indican una gobernabilidad pobre de acuerdo a la valoración de Huther y Shah, a excepción de la municipalidad del Santa que muestra una gobernabilidad media.

\section{REFERENCIAS BIBLIOGRÁFICAS}

Aghón, Gabriel; Alburquerque, Francisco y Cortés, Patricia 2001. Desarrollo Económico Local y Descentralización en América Latina: Un Análisis Comparativo. CEPAL

Díaz, William y Melvy Corredor. 2009. Significación del municipio en el desarrollo local de la República Bolivariana de Venezuela. < http://biblioteca.universia.net/ html_bura/ficha/params/id/42163081.html> [Consultado: 20-05-2014].

Huther, Jeff y Sha Anwar. 2008. Applying a simple measure og good governace to the debate fiscal on fiscal decentralization. Documento de trabajo de investigación., Washington: Banco Mundial.

Instituto Nacional de Estadística. 2014. Resultados ENAHO 2010_2014. Lima.

Ministerio de Economía y Finanzas del Perú MEF. 2014. SIAF amigable. Lima.

Monroy, José; Perez, José; García, David. 2003. Los espacios del poder. Desarrollo local y poder local en los procesos de localización industrial y desarrollo socioeconómico: el caso de Atlacomulco, Estado de México, 1980-2002. México: Investigaciones geográficas.

Oficina Nacional de Procesos Electorales. 2014. Resultados elecciones municipales Perú 2014. Lima. 
Orellana, Esperanza. 2009. El Rol del Municipio en el Desarrollo Local. Colombia: <http://biblioteca.universia.net/html_bura/ficha/params/id/42163081.html> [Consultado: 15-05-2014].

Pontificia Universidad Catolica del Perú. 2010. Indice de competitividad regional del Perú.. Lima: CENTRUM.

Porter, Michael. (2000), Location, competition and economic development: local clusters in the global economy, Economic Development Quarterly, vol. 14, No 1 , Londres: Sage Publications.

Qian, Yingi y Barry. Weingast. 1997. Federalism as a commitment to reserving market incentives. Washington: Economic perspectives.

Vázquez, Antonio; Von Haldenwang, Christian. y Zubieta, Javier. 2001. Desarrollo económico localy descentralización en Latinoamerica. México: CEPAL/GTZ.

Fecha de recepción: 16 de setiembre de 2016

Fecha de aceptación: 10 de noviembre de 2016

\section{Correspondencia}

Guillermo Napoleón Peláez Díaz

Emperador0604@hotmail.com 